\title{
Halogen activation in the plume of Masaya volcano: field observations and box model investigations
}

Julian Rüdiger $^{1, *}$, Alexandra Gutmann ${ }^{1}$, Nicole Bobrowski ${ }^{2,3}$, Marcello Liotta ${ }^{4}$, J. Maarten de Moor $^{5}$, Rolf Sander ${ }^{3}$, Florian Dinger ${ }^{2,3}$, Jan-Lukas Tirpitz ${ }^{2}$, Martha Ibarra ${ }^{6}$, Armando Saballos ${ }^{6}$, María Martínez $^{5}$, Elvis Mendoza ${ }^{6}$, Arnoldo Ferrufino ${ }^{6}$, John Stix $^{7}$, Juan Valdés ${ }^{8}$, Jonathan M. Castro $^{9}$, and Thorsten Hoffmann ${ }^{1}$

${ }^{1}$ Johannes Gutenberg-University, Institute of Inorganic and Analytical Chemistry, Mainz, Germany

${ }^{2}$ Institute for Environmental Physics, University of Heidelberg, Heidelberg, Germany

${ }^{3}$ Max-Planck Institute for Chemistry, Mainz, Germany

${ }^{4}$ Istituto Nazionale di Geofisica e Vulcanologia, Sezione di Palermo, Italy

${ }^{5}$ Observatorio Vulcanológico y Sismológico de Costa Rica Universidad Nacional, Heredia, Costa Rica

${ }^{6}$ Instituto Nicaragüense de Estudios Territoriales, Nicaragua

${ }^{7}$ Department of Earth and Planetary Sciences, McGill University, Montreal, Canada

${ }^{8}$ Laboratorio de Química de la Atmósfera, Universidad Nacional, Heredia, Costa Rica

${ }^{9}$ Institute of Geosciences, Johannes Gutenberg University Mainz, Mainz, Germany

* now at Federal Highway Research Institute, Germany

Correspondence to: Thorsten Hoffmann (t.hoffmann@uni-mainz.de)

Table S 1: Gas species used in the HSC model and the mixing ratio of the atmospheric background composition taken from (Roberts et al., 2014).

\begin{tabular}{|c|c|c|c|}
\hline Species & Mixing ratio & Species & Mixing ratio \\
\hline $\mathrm{H}_{2} \mathrm{O}(\mathrm{g})$ & - & $\mathrm{Cl}(\mathrm{g})$ & - \\
\hline $\mathrm{Ar}(\mathrm{g})$ & 0.0095 & $\mathrm{I}(\mathrm{g})$ & - \\
\hline $\mathrm{N}_{2}(\mathrm{~g})$ & 0.78 & $\mathrm{~F}(\mathrm{~g})$ & - \\
\hline $\mathrm{O}_{2}(\mathrm{~g})$ & 0.21 & $\mathrm{BrO}(\mathrm{g})$ & - \\
\hline $\mathrm{CO}_{2}(\mathrm{~g})$ & 0.0004 & $\mathrm{ClO}(\mathrm{g})$ & - \\
\hline $\mathrm{CH}_{4}(\mathrm{~g})$ & $1.70 \mathrm{E}-06$ & $\mathrm{IO}(\mathrm{g})$ & - \\
\hline $\mathrm{CO}(\mathrm{g})$ & $1.5 \mathrm{E}-07$ & $\mathrm{O}(\mathrm{g})$ & - \\
\hline $\mathrm{O}_{3}(\mathrm{~g})$ & $6.40 \mathrm{E}-08$ & $\mathrm{Cl}(\mathrm{g})$ & - \\
\hline $\mathrm{OH}(\mathrm{g})$ & $6.90 \mathrm{E}-13$ & $\mathrm{Br}(\mathrm{g})$ & - \\
\hline $\mathrm{H}_{2} \mathrm{O}_{2}(\mathrm{~g})$ & - & $\mathrm{I}(\mathrm{g})$ & - \\
\hline $\mathrm{HO}_{2}(\mathrm{~g})$ & $3.00 \mathrm{E}-11$ & $\mathrm{BrCl}(\mathrm{g})$ & - \\
\hline $\mathrm{NO}_{(\mathrm{g})}$ & $5.00 \mathrm{E}-11$ & $\mathrm{HOCl}(\mathrm{g})$ & - \\
\hline $\mathrm{NO}_{2}(\mathrm{~g})$ & $1.10 \mathrm{E}-10$ & $\mathrm{H}_{2}(\mathrm{~g})$ & - \\
\hline $\mathrm{HNO}_{2}(\mathrm{~g})$ & - & $\mathrm{H}(\mathrm{g})$ & - \\
\hline $\mathrm{NO}_{3}(\mathrm{~g})$ & $5.00 \mathrm{E}-14$ & $\mathrm{H}_{2} \mathrm{SO}(\mathrm{g})$ & - \\
\hline $\mathrm{HNO}_{3}(\mathrm{~g})$ & - & $\mathrm{N}_{2} \mathrm{O}(\mathrm{g})$ & - \\
\hline $\mathrm{N}_{2} \mathrm{O}_{5}(\mathrm{~g})$ & - & $\mathrm{HNO}(\mathrm{g})$ & - \\
\hline $\mathrm{CH}_{3} \mathrm{OH}(\mathrm{g})$ & - & $\mathrm{OClO}(\mathrm{g})$ & - \\
\hline $\mathrm{SO}_{2}(\mathrm{~g})$ & - & $\mathrm{HF}(\mathrm{g})$ & - \\
\hline $\mathrm{HCl}(\mathrm{g})$ & - & $\mathrm{HI}(\mathrm{g})$ & - \\
\hline $\mathrm{HBr}(\mathrm{g})$ & - & $\mathrm{Br}(\mathrm{g})$ & - \\
\hline
\end{tabular}



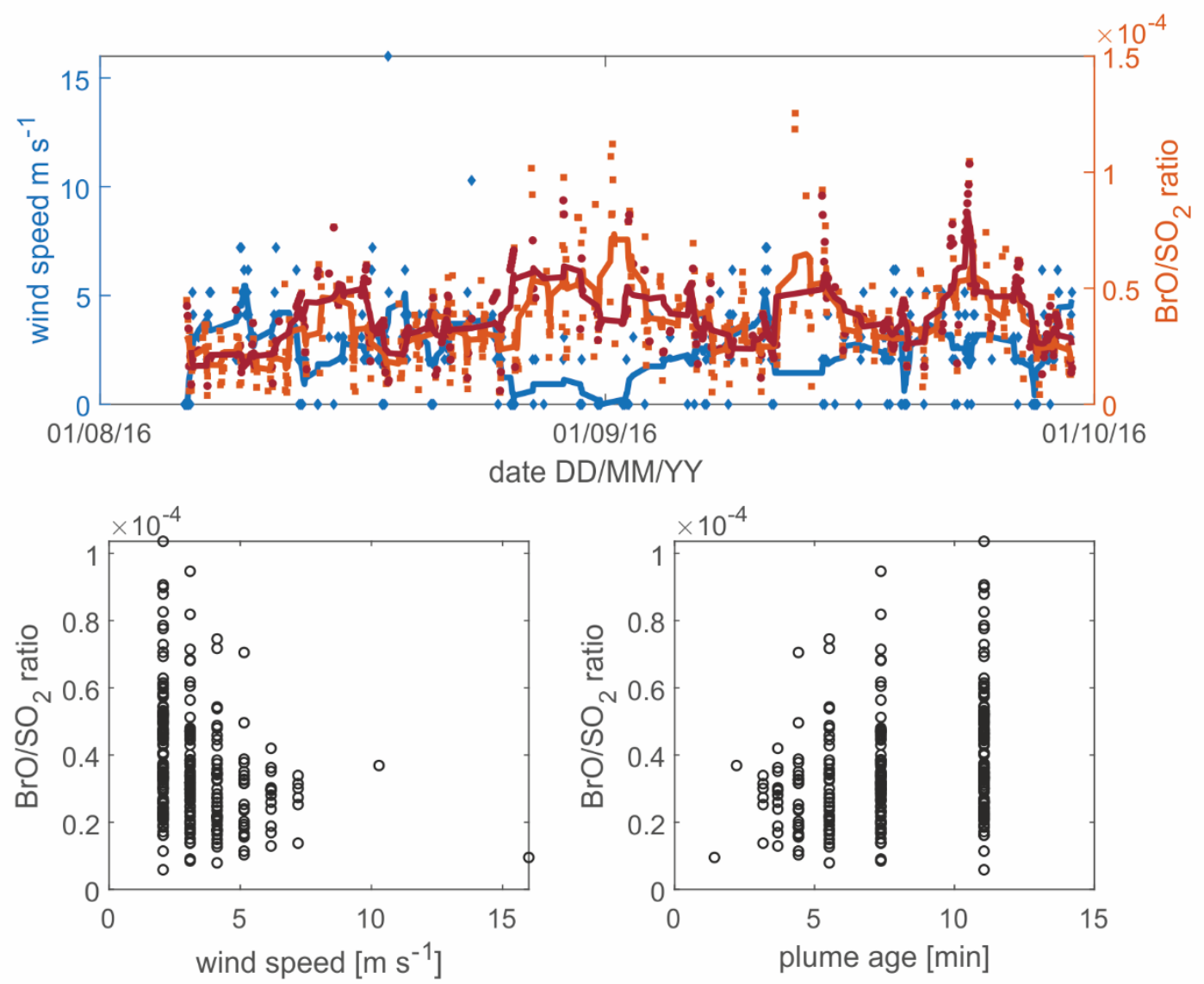

Figure S 1: (a) Wind speed at the meteorological measurement station at Managua airport (blue) and $\mathrm{BrO} \mathrm{SO}_{2}$ mixing ratios at Masaya volcano (orange) and interpolated $\mathrm{BrO} / \mathrm{SO}_{2}$ mixing ratios (red) at the time of wind speed measurements; (b) $\mathrm{BrO} / \mathrm{SO}_{2}$ mixing ratio vs. wind speed and (c) $\mathrm{BrO} / \mathrm{SO}_{2}$ mixing ratio vs. estimated plume age 

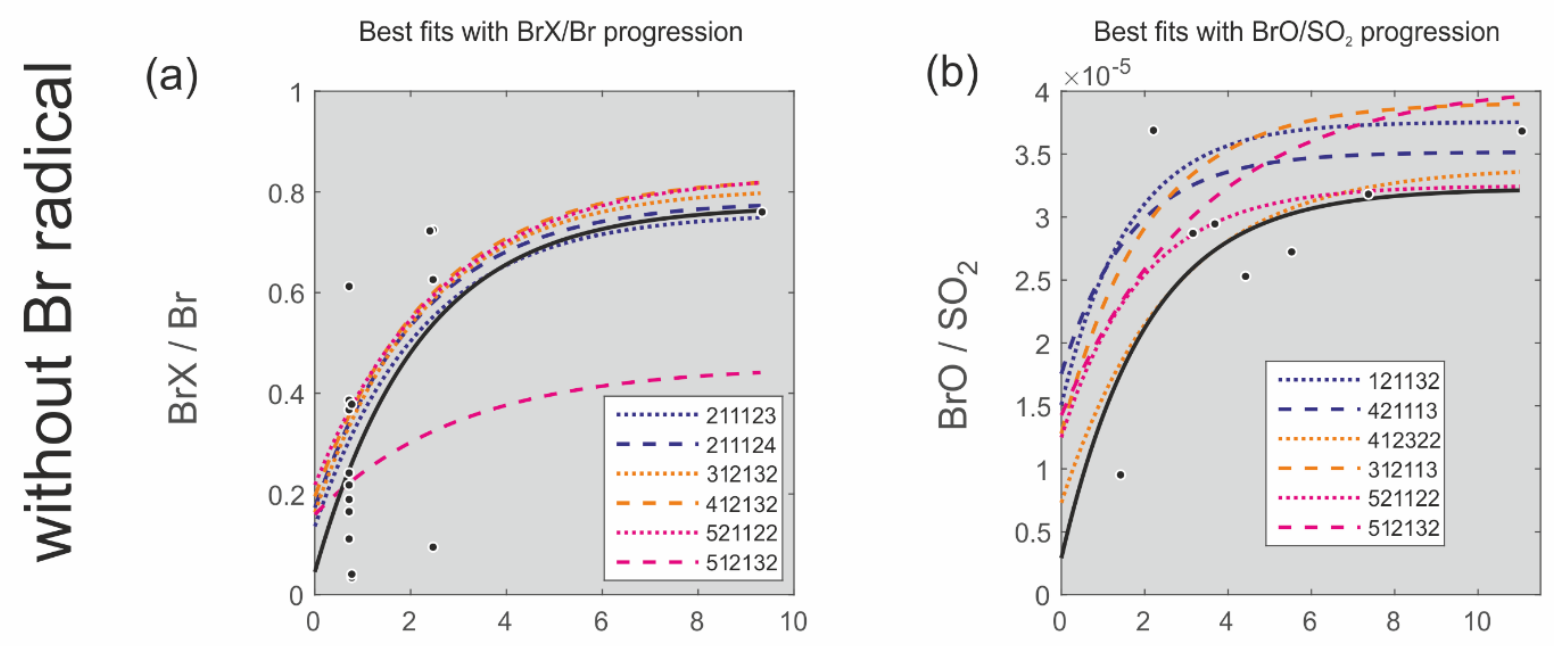

(c)
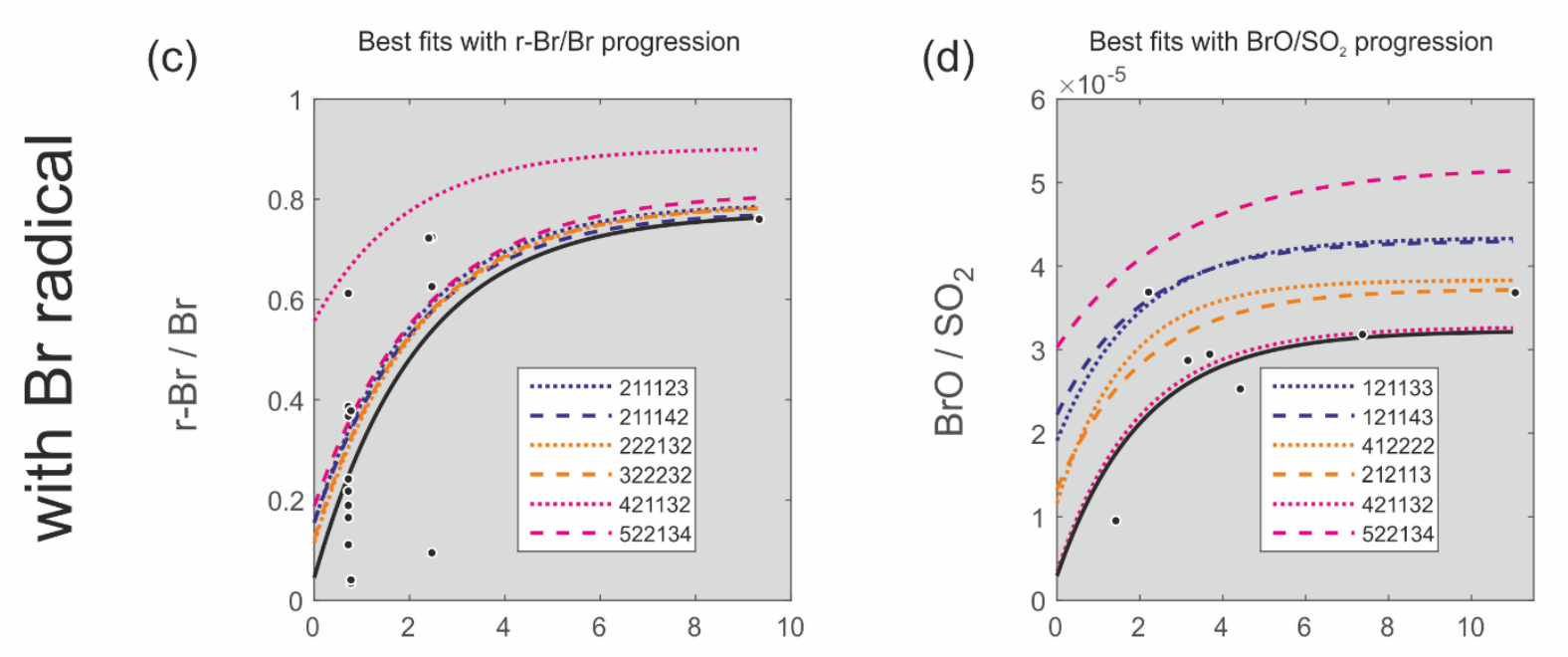

estimated plume age [min]

\begin{tabular}{|c|c|c|c|c|c|c|}
\hline \multicolumn{7}{|c|}{$\begin{array}{l}\text { Best fits of } \mathrm{BrX} / \mathrm{Br} \text { and } \mathrm{BrO} / \mathrm{SO}_{2} \text { progressions - separate } \\
\ldots \ldots . . . . \\
-\ldots .-\end{array}$} \\
\hline \multicolumn{7}{|c|}{6 digit number code of the model runs: } \\
\hline$\#$ & $\mathrm{~V}_{\mathrm{M}}: \mathrm{V}_{\mathrm{A}}$ & $\begin{array}{c}\text { Quenching } \\
\mathrm{SO}_{2}\end{array}$ & $\mathrm{HxO}_{\mathrm{y}}$ & $\begin{array}{l}\text { Dilution } \\
\text { time to } 1 / \mathrm{e}\end{array}$ & $\begin{array}{l}\text { Aerosol number } \\
\text { concentration }\end{array}$ & $\begin{array}{c}\text { Aerosol } \\
\text { particle size }\end{array}$ \\
\hline 1 & $0: 100$ & $6 \mathrm{ppm}$ & air & $10 \mathrm{~min}$ & $1 \times 10^{8} \mathrm{~m}^{-3}$ & $5 \times 10^{-8} \mathrm{~m}$ \\
\hline 2 & 2:98 & $30 \mathrm{ppm}$ & magmatic & $20 \mathrm{~min}$ & $1 \times 10^{9} \mathrm{~m}^{-3}$ & $3 \times 10^{-7} \mathrm{~m}$ \\
\hline 3 & 5:95 & 300 ppm & & $30 \mathrm{~min}$ & $3 \times 10^{9} \mathrm{~m}^{-3}$ & $9 \times 10^{-7} \mathrm{~m}$ \\
\hline 4 & 10:90 & $500 \mathrm{ppm}$ & & & $5 \times 10^{9} \mathrm{~m}^{-3}$ & $1.5 \times 10^{-6} \mathrm{~m}$ \\
\hline 5 & $15: 85$ & 1000 ppm & & & & \\
\hline 6 & $35: 65$ & & & & & \\
\hline 7 & 50:50 & & & & & \\
\hline
\end{tabular}

Figure S 21: (a) BrX/Br samples (black circles), fit through the sample data points using $f(x)=a^{*} \exp \left(-b^{*} x\right)+c$ (Eq. 1, see text) as a fit function (black line), fits of the closest model runs see description in legend (modelled BrX includes: $\mathrm{Br}_{2}, \mathrm{BrCl}, \mathrm{BrNO}_{2}, \mathrm{BrNO}_{3}, \mathrm{HOBr}$ and $\mathrm{BrO}$ ), (b) $\mathrm{BrO} \mathrm{SO}_{2}$ median values for different plume ages and fits through measurement and model data using Eq. 1 similar to (a), (c) and (d) similar procedure as in (a) and (b), but modelled reactive $\mathrm{Br}$ species ( $\mathrm{r}-\mathrm{Br}$ ) also include $\mathrm{Br}$ radicals. 

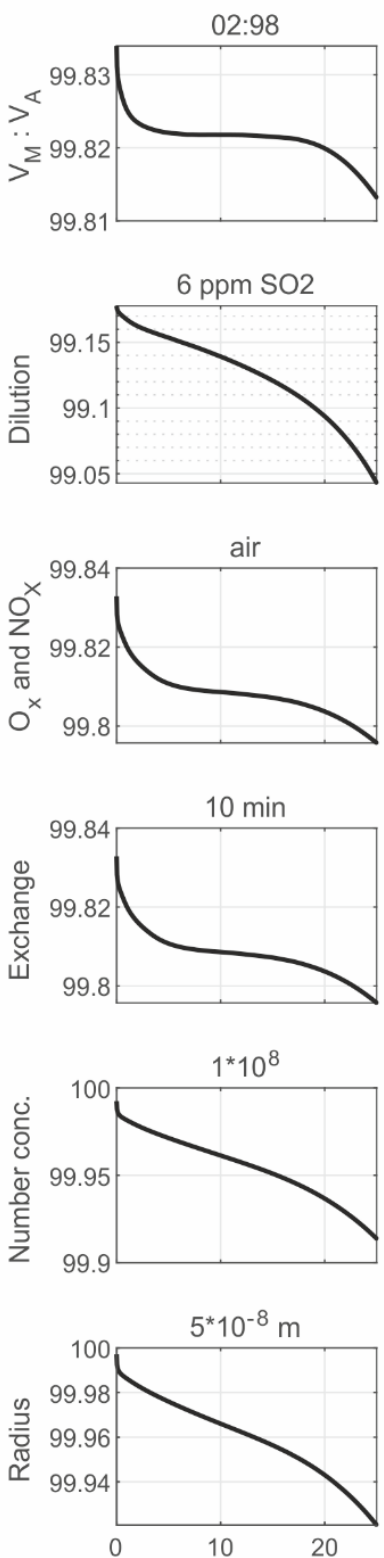

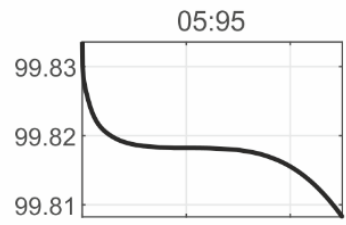

30 ppm SO2
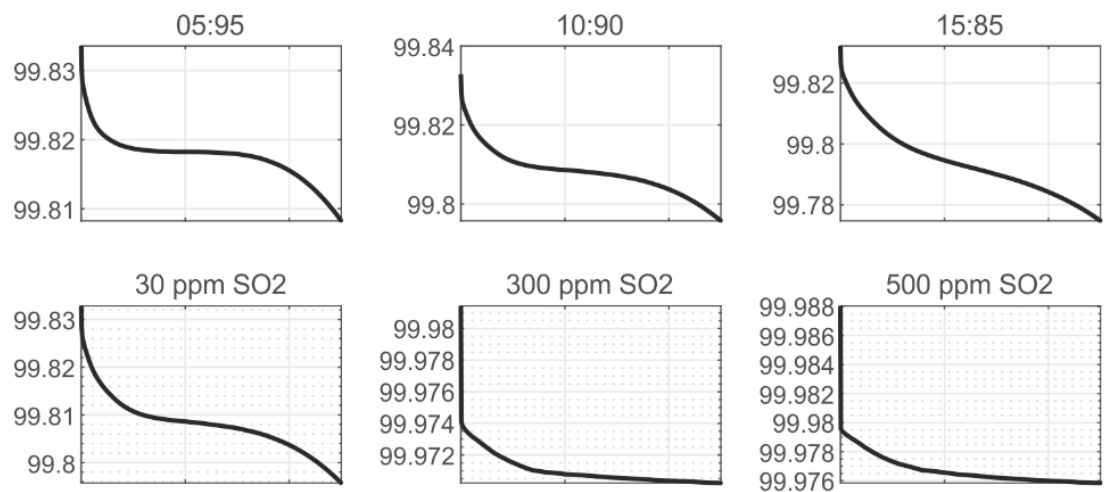

99.976
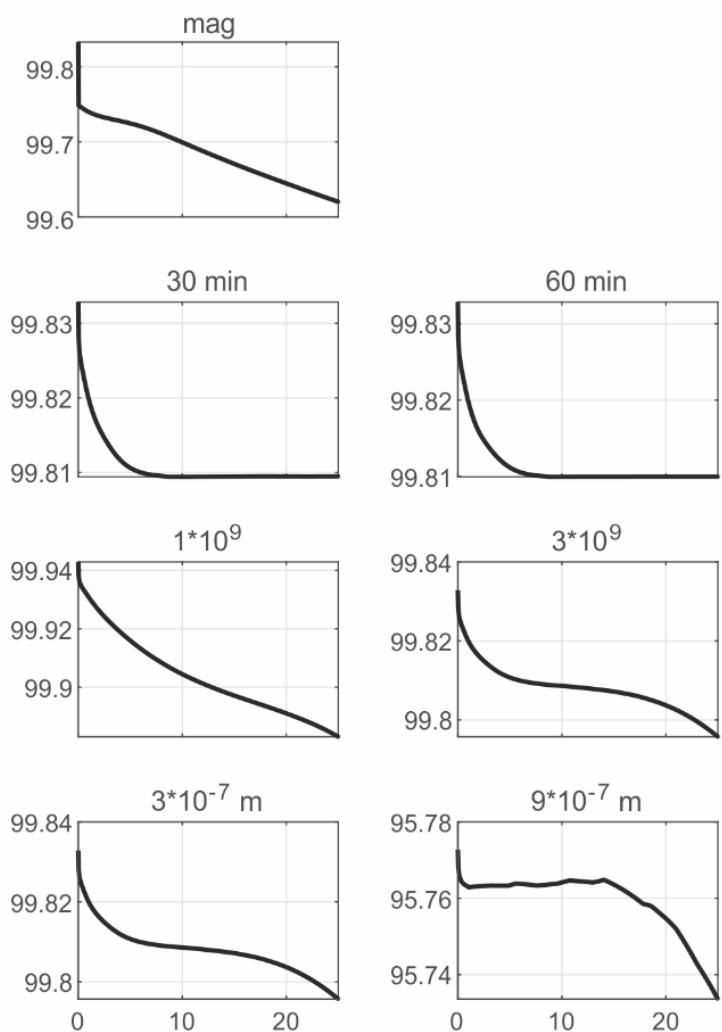
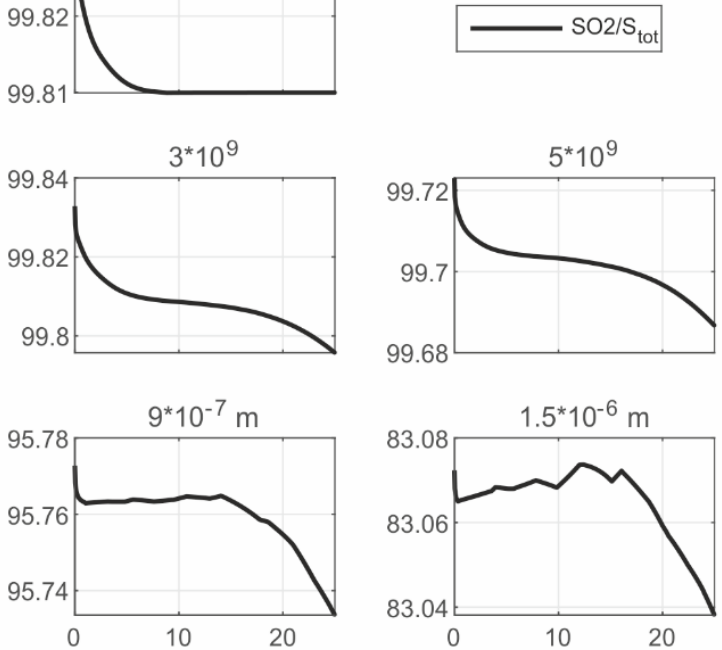

Figure S 2: $\mathrm{SO}_{2}$ over total sulfur for selected CAABA/MECCA model runs corresponding to Figure 9. Variations of the base run by changes of one parameter (horizontal rows). 

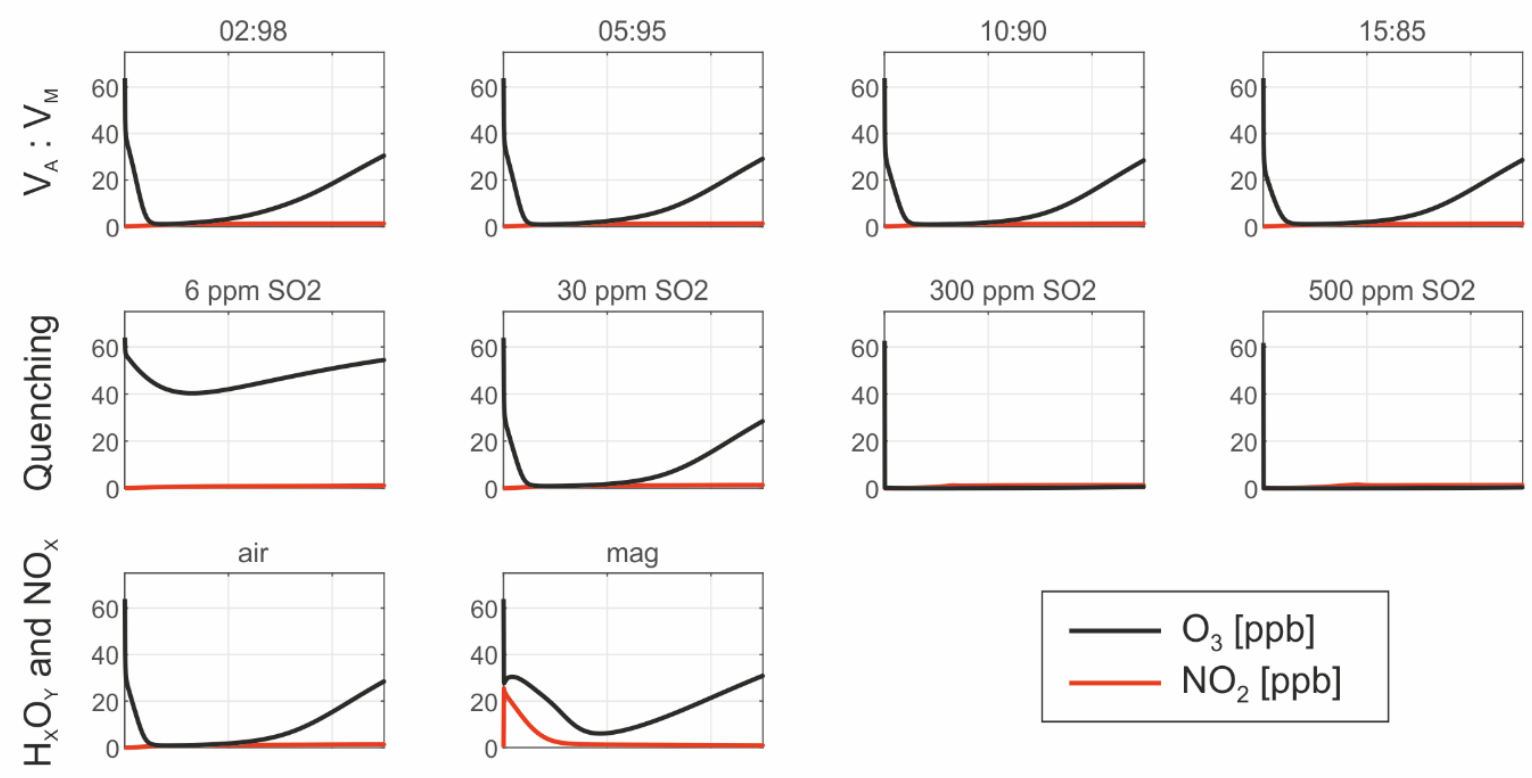

\section{$-\mathrm{O}_{3}$ [ppb] $\mathrm{NO}_{2}[\mathrm{ppb}]$}
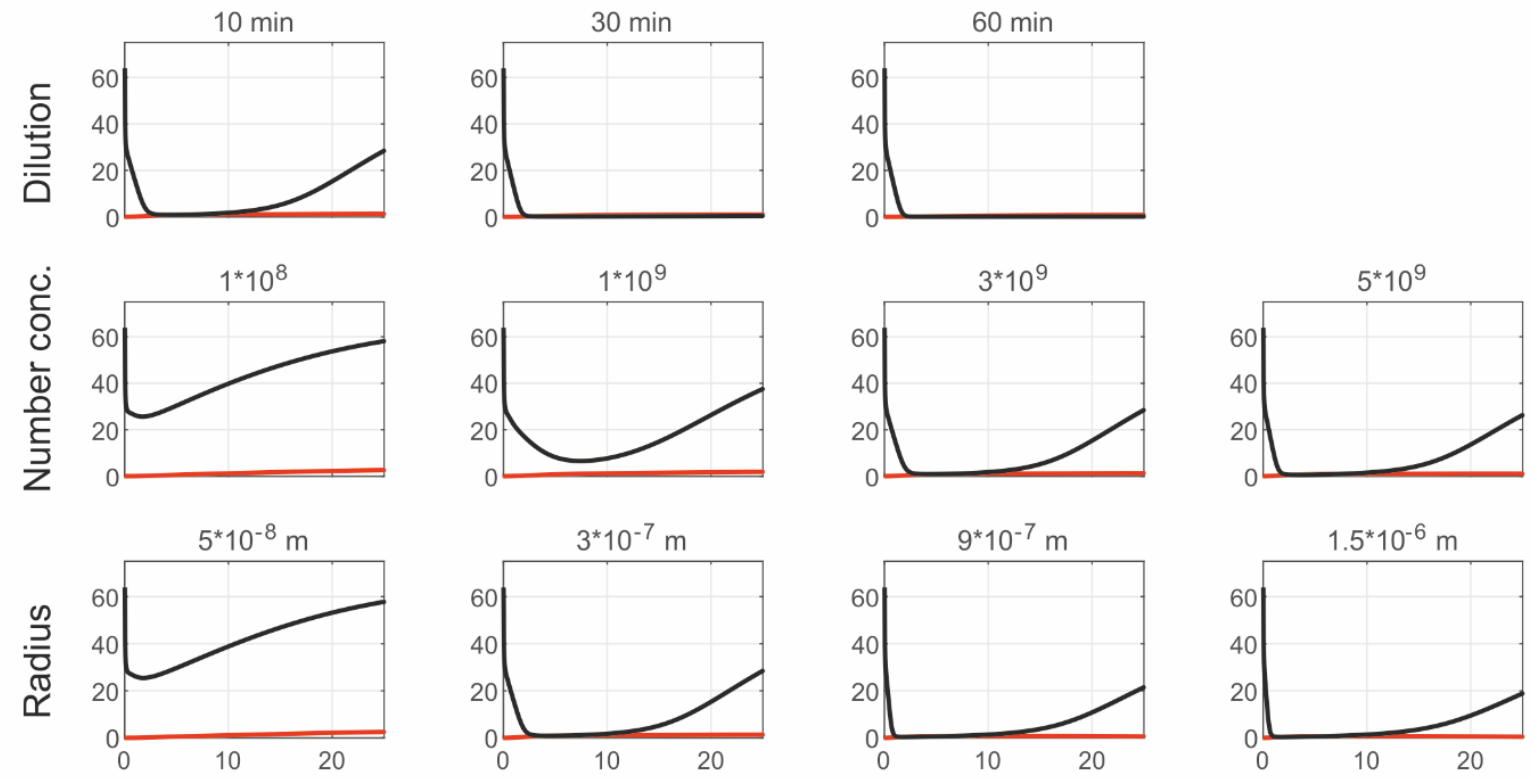

Figure $\mathrm{S} 3: \mathrm{O}_{3}$ and $\mathrm{NO}_{2}$ mixing ratios during the model runs in Figure 9. Variations of the base run by changes of one parameter (horizontal rows) 

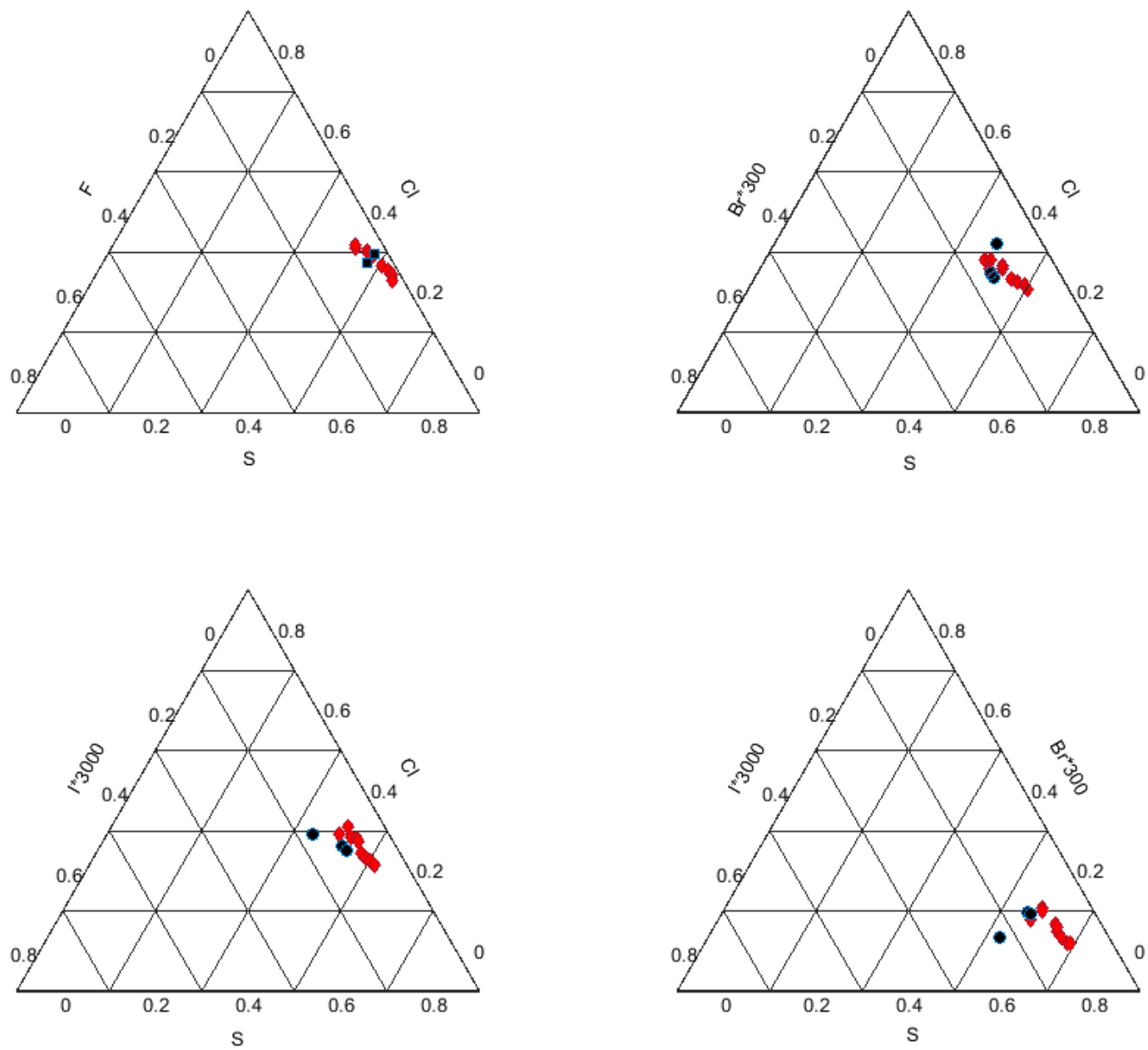

Figure S 4: Ternary plots of Halogen to Sulfur ratios; red diamonds: July samples, black circles: September samples 
Table S 2: Results of denuder, alkaline and electrochemical sensor measurements $(G=$ ground, $R=$ Raschig tube, $D=D r e c h s e l$ bottle, $U A V=$ unmanned aerial vehicle, Type $1=1 \mathrm{M}$ $\mathrm{NaOH}, 4$ = $4 \mathrm{M} \mathrm{NaOH})$. HalX (e.g., $\mathrm{Br}_{2}, \mathrm{Cl}_{2}$ or $\mathrm{BrCl}$ ) mixing ratios were derived from denuder samples with known volume of sampled plume gas; for certain alkaline trap samples the sampled volume is unknown and only a ratio of the respective halogen to sulfur was calculated from sample concentrations.

\begin{tabular}{|c|c|c|c|c|c|c|c|c|c|c|c|c|c|c|c|c|c|c|c|c|c|c|}
\hline No. & Date & Type & Location & \begin{tabular}{|c|}
$\begin{array}{c}\text { Distance } \\
{[\mathrm{m}]}\end{array}$ \\
\end{tabular} & $\begin{array}{l}\mathrm{s}\left(\mathrm{SO}_{2}\right) \\
{[\mathrm{ppm}]}\end{array}$ & F [ppm] & $\mathrm{F} / \mathrm{S}$ & Cl [ppm] & $\mathrm{cl} / \mathrm{s}$ & $\mathrm{CIX}[\mathrm{ppb}]$ & $\mathrm{cl} x / \mathrm{s}$ & $\mathrm{cl} / \mathrm{Cl}$ & $B r_{y}[p p b]$ & $\mathrm{Br} / \mathrm{S}$ & BrX [ppb] & $\mathrm{Br} / \mathrm{S}$ & $\mathrm{Br} / \mathrm{Br}$ & I [ppb] & $1 / 5$ & $\mathrm{IX}[\mathrm{ppb}]$ & $\mathrm{Ix} / \mathrm{s}$ & $\mid x / 1$ \\
\hline 1 & 14.07 .2016 & GR & 1 |Lookout (s) & $231 \pm 20$ & $4.18 \pm 0.22$ & $0.12 \pm 0.01$ & $1.6 \mathrm{E}-01 \pm 1.2 \mathrm{E}-02$ & $3.24 \pm 0.17$ & $0.77 \pm 0.06$ & & & & $3.72 \pm 0.07$ & \begin{tabular}{|l|l}
$8.89 \mathrm{E}-04 \pm 4.95 \mathrm{E}-05$ \\
\end{tabular} & & & & $0.30 \pm 0.07$ & $7.2 E-05 \pm 1.8 \mathrm{E}-05$ & & & \\
\hline 2 & 14.07 .2016 & GD & 1 Pole & $214 \pm 20$ & $0.71 \pm 0.04$ & & $1.1 E-01 \pm 8.1 E-03$ & $0.53 \pm 0.03$ & $0.75 \pm 0.06$ & $0.17 \pm 0.24$ & $2.4 \mathrm{E}-04 \pm 3.4 \mathrm{E}-04$ & $3.2 E-04 \pm 4.6 E-04$ & & $1.57 \mathrm{E}-03 \pm 1.14 \mathrm{E}-04$ & $0.68 \pm 0.15$ & $\begin{array}{ll}5 & 9.6 E-04 \pm 2.2 E-04\end{array}$ & $0.61 \pm 0.14$ & & & $0.11 \pm 0.01$ & $1.6 \mathrm{E}-04 \pm 2.0 \mathrm{E}-05$ & \\
\hline 3 & 14.07.2016 & & 1 Pole & $214 \pm 20$ & & $0.15 \pm 0.01$ & $1.8 \mathrm{E}-01 \pm 1.3 \mathrm{E}-02$ & $2.83 \pm 0.15$ & $0.80 \pm 0.06$ & & & & $3.54 \pm 0.06$ & $1.00 \mathrm{E}-03 \pm 5.48 \mathrm{E}-05$ & & & & 0.06 & $5.2 \mathrm{E}-0.5$ & & & \\
\hline \begin{tabular}{|l|l}
4 \\
\end{tabular} & 14.07 .2016 & $6 D$ & 1 Pole & 21 & $0.83 \pm 0.04$ & & $5.6 E-02 \pm$ & $0.63 \pm 0.03$ & $0.76 \pm 0.06$ & $0.17 \pm 0.24$ & $2.1 E-04 \pm 3.0 E-04$ & $2.7 \mathrm{Z}-04 \pm 3.9 \mathrm{E}-04$ & $1.85 \pm 0.08$ & $2.24 \mathrm{E}-03 \pm 1.50 \mathrm{E}-04$ & $0.68 \pm 0.15$ & \begin{tabular}{c|c|}
5 & $8.2 E-04 \pm 1.9 E-04$
\end{tabular} & $0.37 \pm 0$. & & $5.66-05 \pm$ & $0.11 \pm 0.01$ & $1.4 E-04 \pm 1.7 E-05$ & \\
\hline 5 & 14.07.2016 & & 4 |Pole & $214 \pm 20$ & & $0.52 \pm 0.03$ & $1.2 \mathrm{E}-01 \pm 8.8 \mathrm{E}-03$ & & $0.90 \pm 0.06$ & & & & & & & & & & & & & \\
\hline 6 & 15.07.2016 & & 1 Nindi & 74 & 06 & $0.34 \pm 0.02$ & $3.8 \mathrm{E}-02 \pm 2.7 \mathrm{E}-03$ & \pm 0.03 & 0. & $3 \pm 0.18$ & $6.5 \mathrm{E}-04 \pm 1.6 \mathrm{E}-04$ & $1.3 \mathrm{E}-03 \pm 3.3 \mathrm{E}-04$ & 0.03 & $6.86 \mathrm{E}-04 \pm 4.711 \mathrm{E}-05$ & $0.56 \pm 0.10$ & \begin{tabular}{c|c}
$0.0 \mathrm{E}-04 \pm 8.9 \mathrm{E}-05$ \\
\end{tabular} & $0.72 \pm 0.13$ & $0.05 \pm 0.03$ & $4.7 E-05 \pm 3.1 \mathrm{E}-05$ & $0.07 \pm 0.6$ & $5.9 E-05 \pm 7.0 \mathrm{E}-06$ & \\
\hline \begin{tabular}{|l|}
7 \\
8
\end{tabular} & $\begin{array}{l}15.07 .2016 \\
15072016\end{array}$ & $\begin{array}{l}6 R \\
G D \\
G R\end{array}$ & $\begin{array}{l}1 \text { Pole } \\
\text { 4Pole }\end{array}$ & & $8.81 \pm 0.46$ & & & $4.45 \pm 0.23$ & $\begin{array}{l}0.51 \pm 0.04 \\
0.72 \pm 0.05\end{array}$ & $0.26 \pm 0.26$ & $3.0 \mathrm{E}-05 \pm 3.0 \mathrm{E}-05$ & $5.9 E-05 \pm 5.9 E-05$ & $4.44 \pm 0.08$ & 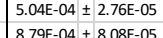 & $1.71 \pm 0.19$ & 9 | $1.9 \mathrm{EE}-04 \pm 2.4 \mathrm{E}-05$ & $0.39 \pm 0.04$ & $0.33 \pm 0.08$ & $\begin{array}{l}3.8 E-05 \pm 8.8 E-06 \\
7 \\
72 E-05 \pm 25-05\end{array}$ & $50.17 \pm 0.02$ & $1.9 E-05 \pm 2.1 E-06$ & $0.51 \pm 0.13$ \\
\hline \begin{tabular}{|c|c}
8 \\
9
\end{tabular} & $\begin{array}{l}13.0 \\
15.0\end{array}$ & GD & $\begin{array}{l}4 \text { Pole } \\
4 \text { Pole }\end{array}$ & \begin{tabular}{|l|}
$214 \pm 20$ \\
$214 \pm 20$
\end{tabular} & & & & & $0.84 \pm 0.06$ & & & & & 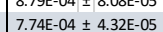 & & & & & 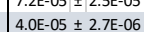 & & & \\
\hline 10 & & & 1 Nindi & & \pm 0.05 & $0.37 \pm 0.02$ & $4.9 E-02 \pm 3.5 E-03$ & 07 & & & & & 0.04 & 1.6 & 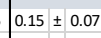 & $6 E-04 \pm 6$. & $0.09 \pm 0.04$ & 1.04 & & $0.03 \pm 0.00$ & $-05 \pm 4.3 E-06$ & 5 \\
\hline \begin{tabular}{|l|l|}
11 \\
\end{tabular} & 16.07.2016 & & 1 Pole & 0 & \begin{tabular}{|l|l|}
$7.56 \pm 0.39$ \\
\end{tabular} & & & $4 \pm 0.24$ & $0.60 \pm 0.04$ & & & & $4.97 \pm 0.10$ & $6.57 E_{-04} \pm$ & & & & & & & & \\
\hline 12 & & UAV & & 198 & 1. & & & & & & & & & & $1.26 \pm 0.69$ & $\begin{array}{l}9.6 \mathrm{E}-04 \pm 3.6 \mathrm{E}-04 \\
\end{array}$ & & & & & & \\
\hline 13 & & & 1| Nindit & & & $0.10 \pm 0.01$ & FE-02 $\pm 1.98-03$ & & & & & & & 1. & & & & & & & & \pm 0.30 \\
\hline 14 & 18.07 .20 & 5 GR & 1 Pole & & $3.61 \pm 0.19$ & & & $1.95 \pm 0.10$ & $0.54 \pm 0.04$ & & & & & & $0.45 \pm 0.0$ & & 0.03 & & & & & \\
\hline 15 & 20.07 .20 & & 11Pole & 214 & $2.65 \pm 0.14$ & $0.09 \pm 0.01$ & $3 E-02 \pm 2.3 E-03$ & $1.51 \pm 0.08$ & $0.57 \pm 0.04$ & & & & $1.55 \pm 0.03$ & $5.84 E-04 \pm$ & $0.34 \pm 0.05$ & \begin{tabular}{l|l}
5 & $1.3 \mathrm{E}-04 \pm 2.1 \mathrm{E}-05$
\end{tabular} & $0.22 \pm 0.03$ & $0.12 \pm 0.03$ & $4.5 E-0$ & & & \\
\hline 16 & & GR & 1 Nind & & $0.96 \pm 0.05$ & & & $0.89 \pm 0.05$ & $0.93 \pm 0.07$ & & & & $1.22 \pm 0.03$ & $1.28 \mathrm{E}-03 \pm 7.30 \mathrm{E}-05$ & & & & $0.06 \pm 0.03$ & $6.6 E-05 \pm 3.0 E-05$ & & & $0.31 \pm 0.15$ \\
\hline 17 & $20.07 .2 \mathrm{~F}$ & Uav & Calde & 100 & $1 \pm 0.05$ & & & & & & & & & & $2.91=$ & -03 & & & & & & \\
\hline 18 & 20.07.2016 & UAV & & $2 \pm 100$ & $59 \pm 0.05$ & & & & & & & & & & $1.19 \pm$ & \begin{tabular}{l|l|l}
2 & $2.0 E-03 \pm 1.1 E-03$
\end{tabular} & & & & $0.06 \pm 0.03$ & $1.1 E-c$ & \\
\hline 19 & 21.07.2016 & GR & 1 Nind & $740 \pm 50$ & $74 \pm 0.14$ & $0.29 \pm 0.01$ & -03 & 10 & $3 \pm 0.05$ & & & & .06 & 9. & & & & & & & & \\
\hline 20 & 21.07. & SGR & 1/Nindir & & 08 & $0.56 \pm 0.03$ & $02 \pm 5.1 E-03$ & $5 \pm 0.08$ & $0.91 \pm 0.07$ & & & & & 1.12 & & & & & & & & \\
\hline 21 & & 5 GR & 11Pole & & $4.06 \pm 0.21$ & & $1.1-01 \pm$ & $2.76 \pm 0.14$ & 0.68 & & & & & 7.5 & & & & & & & & \\
\hline 22 & 21.07. & 5 GR & 11Pole & 2 & $7.67 \pm 0.40$ & & 1.11E-01 \pm 7 & $5.36 \pm 0.28$ & 0.70 & & & & $5.57 \pm 0.55$ & $7.26 \mathrm{E}-\mathrm{T}$ & $0.92 \pm 0.08$ & \begin{tabular}{c|c}
$81.2 \mathrm{E}-04$ & $\pm 1.2 \mathrm{E}-05$
\end{tabular} & $0.17 \pm 0.02$ & $0.34 \pm 0.55$ & $4.4 E-$ & $0.11 \pm 0.01$ & $1.4 E-05 \pm 1.2 E-06$ & $0.32 \pm 0.52$ \\
\hline 23 & 21.07.2016 & $56 D$ & 11Pole & $214 \pm 20$ & & $0.20 \pm$ & $7.4 E-02 \pm 5$ & & $0.63 \pm 0.04$ & & & & & $6.16 \mathrm{E}-04 \pm$ & & & & & $4.9 E-0.5$ & & & \\
\hline 24 & 21.07.2016 & 5 GD & 11Pole & 214 & & $0.14 \pm 0.01$ & $8.4 \mathrm{E}-02 \pm 6.0 \mathrm{EE}-03$ & & $0.84 \pm 0.06$ & & & & & $1.24 \mathrm{E}-03 \pm 6$ & & & & & 9.2E- & & & \\
\hline 25 & 22.07.2016 & 5 GR & 1 1 Look & & $\mathrm{b1} \pm 0.31$ & $0.46 \pm 0.02$ & $7.6 \mathrm{E}-02 \pm 5.4 \mathrm{E}-03$ & $4.34 \pm 0.23$ & $0.72 \pm 0.05$ & & & & $5.86 \pm 0.11$ & $9.75 \mathrm{E}-04 \pm 5$ & 0.20 & 73.3 & & $0.32 \pm 0.11$ & 5.3E-05 & & $878-7$ & \\
\hline 26 & 22.07.2 & FR & 1|Looko & 20 & $6.01 \pm 0.31$ & & & $4.34 \pm 0.23$ & $0.72 \pm 0.05$ & & & & & $9.75 E-04 \pm$ & & & & $0.32 \pm 0.11$ & $5.3 E-05$ & & & \\
\hline 27 & 01.09 .2 & 5 GR & 1|Pole & 214 & $5.16 \pm 0.27$ & $0.52 \pm 0.03$ & $\mid 1.0 \mathrm{E}-01 \pm 7.2 \mathrm{E}-03$ & $\mid 3.35 \pm 0.17$ & $0.65 \pm 0.05$ & & 2.2E-04 $\pm 4.3 \mathrm{E}-05$ & $3.5 \mathrm{E}-04 \pm 6.5 \mathrm{E}-05$ & $4.97 \pm 0.08$ & $9.63 \mathrm{E}-04 \pm 5.27 \mathrm{E}-05$ & & \begin{tabular}{c|c}
$01.8 \mathrm{E}-04$ & $2.2 \mathrm{E}-05$
\end{tabular} & & $0.36 \pm 0.08$ & $7.0 \mathrm{E}-05 \pm 1.7 \mathrm{E}-05$ & $\mid 0.12 \pm 0.01$ & $2.3 E-05 \pm 2.1 \mathrm{EE}-06$ & 08 \\
\hline 28 & 02.09 .2 & SR & 1 | $\operatorname{san} P$ & 720 & & & & & & $0.07 \pm 0.10$ & & & $0.35 \pm 0.02$ & & & & 0.72 & $0.05 \pm 0.02$ & & & & 0.15 \\
\hline 29 & 03.09. & SR & 1| Nindir & 74 & $22 \pm 0.01$ & $0.17 \pm 0.01$ & $5.0 \mathrm{E}-02 \pm 3.6 \mathrm{E}-03$ & $0.64 \pm 0.03$ & $2.94 \pm 0.22$ & $0.55 \pm 0.12$ & $2.5 \mathrm{E}$ & $8.5 E$ & $0.75 \pm 0.02$ & $3.45-03 \pm 2$ & 0.47 & 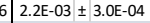 & $0.63 \pm 0.08$ & $0.05 \pm 0.02$ & $2.4 E-04=$ & & & $0.17 \pm 0.09$ \\
\hline 30 & 03. & 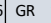 & 1) Pole & & & & & $2.27 \pm 0.12$ & $0.69 \pm 0.05$ & & & $9 \mathrm{gE}-05 \pm 5$ & $3.27 \pm 0.26$ & $9.89 \mathrm{E}-04 \pm 9.2$ & & & $0.11 \pm 0.02$ & $0.24 \pm 0.26$ & $7.3 \mathrm{E}-05 \pm 7.7 \mathrm{E}-00$ & $0.06 \pm 0.00$ & $=06$ & 0.25 \\
\hline 31 & 03 & SUAv & & & & & & & & 1.42 & & & & & & & & & & & & \\
\hline 32 & & sua & & & $14 \pm 0.29$ & & & & & $2.48 \pm 1.35$ & $4 \pm 1.2 \mathrm{E}-04$ & & & & & & & & & & & \\
\hline 3 & & JaV & & & & & & & & & & & & & & & & & & & & \\
\hline 34 & & FR & & & & & & & & & & & & & & & & & & & & \\
\hline $\begin{array}{l}35 \\
36\end{array}$ & $\mid \begin{array}{l}\mid 04.099 .2016 \\
05.05 .9016\end{array}$ & $\begin{array}{l}56 \mathrm{GR} \\
5 \mathrm{GR}\end{array}$ & $\begin{array}{l}1 \\
11 \text { Lookout (night) } \\
1 \text { Cerro Ventarón }\end{array}$ & $\left|\begin{array}{r}231 \pm 20 \\
2800 \pm 200\end{array}\right|$ & $0.85 \pm 0.04$ & & & $0.75 \pm 0.04$ & $0.88 \pm 0.06$ & $\begin{array}{l}7.58 \pm 0.83 \\
0.75 \pm 0.13\end{array}$ & $8.9 E-03 \pm 1.1 \mathrm{E}-03$ & $1.0 \mathrm{E}-02 \pm 1.2 \mathrm{E}-03$ & $\mid \begin{array}{l}0.60 \pm 0.06 \\
0.29 \pm 0.02\end{array}$ & $7.01 E-04 \pm 7.59 E_{-}$ & $\left\{\begin{array}{l}0.23 \pm 0.07 \\
0.22 \pm 0.05\end{array}\right.$ & 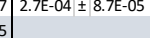 & $\left\{\begin{array}{l}0.38 \pm 0.13 \\
0.76 \pm 0.20\end{array}\right.$ & $\begin{array}{l}0.11 \pm 0.06 \\
0.03 \pm 0.02\end{array}$ & $1.2 \mathrm{E}-04 \pm 6.7 \mathrm{E}-$ & $5\left|\begin{array}{l}0.04 \pm 0.00 \\
0.02 \pm 0.00\end{array}\right|$ & & $\mid \begin{array}{l}0.35 \pm 0.19 \\
0.92 \pm 0.67\end{array}$ \\
\hline
\end{tabular}


Table S 3: Overview on the HSC output and species and their mixing ratios at different volume ratios of atmospheric and magmatic gas at $1000^{\circ} \mathrm{C}$

\begin{tabular}{|c|c|c|c|c|c|c|c|c|}
\hline Temperature & $1000^{\circ} \mathrm{C}$ & & & & & & & \\
\hline VA:VM & $0: 100$ & $1: 99$ & 2:98 & 5:95 & $10: 90$ & $15: 85$ & $35: 65$ & $50: 50$ \\
\hline $\mathrm{H} 2 \mathrm{O}(\mathrm{g})$ & 9.30E-01 & $9.21 \mathrm{E}-01$ & $9.11 \mathrm{E}-01$ & 8.83E-01 & 8.37E-01 & $7.90 \mathrm{E}-01$ & $3.25 \mathrm{E}-01$ & 4.65E-01 \\
\hline N2(g) & $0.00 \mathrm{E}+00$ & $7.80 \mathrm{E}-03$ & $1.56 \mathrm{E}-02$ & $3.90 \mathrm{E}-02$ & $7.80 \mathrm{E}-02$ & 1.17E-01 & 5.07E-01 & 3.90E-01 \\
\hline $\mathrm{O} 2(\mathrm{~g})$ & $6.43 \mathrm{E}-06$ & $2.04 \mathrm{E}-03$ & 4.12E-03 & $1.04 \mathrm{E}-02$ & $2.08 \mathrm{E}-02$ & $3.13 \mathrm{E}-02$ & 1.37E-01 & $1.05 \mathrm{E}-01$ \\
\hline $\mathrm{CO} 2(\mathrm{~g})$ & 4.39E-02 & 4.34E-02 & $4.30 \mathrm{E}-02$ & 4.17E-02 & $3.95 \mathrm{E}-02$ & $3.74 \mathrm{E}-02$ & $1.56 \mathrm{E}-02$ & $2.21 \mathrm{E}-02$ \\
\hline $\mathrm{CH} 4(\mathrm{~g})$ & 1.37E-24 & $1.32 \mathrm{E}-29$ & $3.14 \mathrm{E}-30$ & $4.52 \mathrm{E}-31$ & $9.53 \mathrm{E}-32$ & $3.56 \mathrm{E}-32$ & $1.32 \mathrm{E}-34$ & $6.51 \mathrm{E}-34$ \\
\hline $\mathrm{CO}(\mathrm{g})$ & $1.53 \mathrm{E}-06$ & $8.48 \mathrm{E}-08$ & $5.92 \mathrm{E}-08$ & $3.62 \mathrm{E}-08$ & $2.42 \mathrm{E}-08$ & $1.87 \mathrm{E}-08$ & 3.73E-09 & $6.04 \mathrm{E}-09$ \\
\hline $\mathrm{O} 3(\mathrm{~g})$ & $6.55 \mathrm{E}-18$ & $3.72 \mathrm{E}-14$ & $1.07 \mathrm{E}-13$ & $4.25 \mathrm{E}-13$ & $1.21 \mathrm{E}-12$ & $2.23 \mathrm{E}-12$ & $2.03 \mathrm{E}-11$ & $1.36 \mathrm{E}-11$ \\
\hline $\mathrm{CH} 3 \mathrm{OH}(\mathrm{g})$ & 1.14E-24 & $1.95 \mathrm{E}-28$ & $6.61 \mathrm{E}-29$ & $1.51 \mathrm{E}-29$ & $4.51 \mathrm{E}-30$ & $2.07 \mathrm{E}-30$ & $1.60 \mathrm{E}-32$ & $6.91 \mathrm{E}-32$ \\
\hline $\mathrm{SO} 2(\mathrm{~g})$ & 1.49E-02 & $1.47 \mathrm{E}-02$ & $1.45 \mathrm{E}-02$ & $1.40 \mathrm{E}-02$ & $1.31 \mathrm{E}-02$ & $1.24 \mathrm{E}-02$ & $4.95 \mathrm{E}-03$ & $7.12 \mathrm{E}-03$ \\
\hline $\mathrm{HCl}(\mathrm{g})$ & $1.03 \mathrm{E}-02$ & $1.02 \mathrm{E}-02$ & $1.01 \mathrm{E}-02$ & $9.76 \mathrm{E}-03$ & $9.25 \mathrm{E}-03$ & 8.73E-03 & $3.59 \mathrm{E}-03$ & $5.13 \mathrm{E}-03$ \\
\hline $\mathrm{HBr}(\mathrm{g})$ & $1.06 \mathrm{E}-05$ & $9.11 \mathrm{E}-06$ & $8.75 \mathrm{E}-06$ & 8.07E-06 & $7.24 \mathrm{E}-06$ & $6.59 \mathrm{E}-06$ & $1.98 \mathrm{E}-06$ & $3.16 \mathrm{E}-06$ \\
\hline $\mathrm{HF}(\mathrm{g})$ & $1.05 \mathrm{E}-03$ & $1.04 \mathrm{E}-03$ & $1.03 \mathrm{E}-03$ & $9.99 \mathrm{E}-04$ & $9.46 \mathrm{E}-04$ & 8.93E-04 & $3.68 \mathrm{E}-04$ & $5.26 \mathrm{E}-04$ \\
\hline $\mathrm{HI}(\mathrm{g})$ & $3.22 \mathrm{E}-08$ & 7.64E-09 & $6.30 \mathrm{E}-09$ & $4.76 \mathrm{E}-09$ & 3.67E-09 & $3.03 \mathrm{E}-09$ & $5.58 \mathrm{E}-10$ & $1.01 \mathrm{E}-09$ \\
\hline $\mathrm{Br}(\mathrm{g})$ & 4.77E-07 & $1.75 \mathrm{E}-06$ & $2.01 \mathrm{E}-06$ & 2.37E-06 & 2.61E-06 & $2.70 \mathrm{E}-06$ & $1.83 \mathrm{E}-06$ & $2.28 \mathrm{E}-06$ \\
\hline $\mathrm{Cl}(\mathrm{g})$ & $1.42 \mathrm{E}-06$ & 5.95E-06 & 7.06E-06 & 8.74E-06 & $1.01 \mathrm{E}-05$ & $1.09 \mathrm{E}-05$ & $1.01 \mathrm{E}-05$ & $1.13 \mathrm{E}-05$ \\
\hline I(g) & $6.57 \mathrm{E}-07$ & $6.62 \mathrm{E}-07$ & $6.54 \mathrm{E}-07$ & $6.31 \mathrm{E}-07$ & $5.95 \mathrm{E}-07$ & $5.60 \mathrm{E}-07$ & $2.33 \mathrm{E}-07$ & $3.30 \mathrm{E}-07$ \\
\hline$F(g)$ & $6.34 \mathrm{E}-13$ & $2.66 \mathrm{E}-12$ & $3.16 \mathrm{E}-12$ & $3.92 \mathrm{E}-12$ & $4.54 \mathrm{E}-12$ & $4.88 \mathrm{E}-12$ & $4.53 \mathrm{E}-12$ & 5.07E-12 \\
\hline $\mathrm{BrO}(\mathrm{g})$ & $1.66 \mathrm{E}-12$ & $1.09 \mathrm{E}-10$ & $1.78 \mathrm{E}-10$ & $3.33 \mathrm{E}-10$ & $5.17 \mathrm{E}-10$ & $6.58 \mathrm{E}-10$ & $9.29 \mathrm{E}-10$ & $1.02 \mathrm{E}-09$ \\
\hline $\mathrm{ClO}(\mathrm{g})$ & $1.13 \mathrm{E}-10$ & $8.45 \mathrm{E}-09$ & $1.43 \mathrm{E}-08$ & $2.80 \mathrm{E}-08$ & 4.59E-08 & $6.05 \mathrm{E}-08$ & 1.17E-07 & $1.15 \mathrm{E}-07$ \\
\hline $10(\mathrm{~g})$ & $1.39 \mathrm{E}-12$ & $2.49 \mathrm{E}-11$ & $3.49 \mathrm{E}-11$ & $5.35 \mathrm{E}-11$ & 7.14E-11 & $8.25 \mathrm{E}-11$ & $7.15 \mathrm{E}-11$ & $8.89 \mathrm{E}-11$ \\
\hline $\mathrm{Cl} 2(\mathrm{~g})$ & 2.12E-08 & $3.75 \mathrm{E}-07$ & $5.28 \mathrm{E}-07$ & 8.09E-07 & $1.09 \mathrm{E}-06$ & $1.26 \mathrm{E}-06$ & $1.08 \mathrm{E}-06$ & $1.35 \mathrm{E}-06$ \\
\hline $\mathrm{Br} 2(\mathrm{~g})$ & 4.34E-11 & $5.84 \mathrm{E}-10$ & $7.71 \mathrm{E}-10$ & 1.07E-09 & $1.29 \mathrm{E}-09$ & 1.39E-09 & $6.35 \mathrm{E}-10$ & $9.92 \mathrm{E}-10$ \\
\hline $12(\mathrm{~g})$ & 2.62E-12 & $2.65 \mathrm{E}-12$ & $2.59 \mathrm{E}-12$ & $2.41 \mathrm{E}-12$ & $2.14 \mathrm{E}-12$ & $1.90 \mathrm{E}-12$ & $3.27 \mathrm{E}-13$ & $6.60 \mathrm{E}-13$ \\
\hline $\mathrm{BrCl}(\mathrm{g})$ & 2.05E-09 & $3.16 \mathrm{E}-08$ & $4.31 \mathrm{E}-08$ & $6.30 \mathrm{E}-08$ & 8.01E-08 & 8.93E-08 & $5.59 \mathrm{E}-08$ & $7.81 \mathrm{E}-08$ \\
\hline $\mathrm{HOCl}(\mathrm{g})$ & $6.56 \mathrm{E}-09$ & $1.16 \mathrm{E}-07$ & $1.63 \mathrm{E}-07$ & $2.50 \mathrm{E}-07$ & $3.36 \mathrm{E}-07$ & $3.89 \mathrm{E}-07$ & $3.34 \mathrm{E}-07$ & 4.17E-07 \\
\hline $\mathrm{H} 2(\mathrm{~g})$ & $1.91 \mathrm{E}-05$ & $1.06 \mathrm{E}-06$ & $7.41 \mathrm{E}-07$ & $4.52 \mathrm{E}-07$ & $3.03 \mathrm{E}-07$ & $2.33 \mathrm{E}-07$ & $4.59 \mathrm{E}-08$ & 7.50E-08 \\
\hline $\mathrm{H}(\mathrm{g})$ & 3.16E-09 & $7.44 \mathrm{E}-10$ & $6.21 \mathrm{E}-10$ & $4.86 \mathrm{E}-10$ & $3.97 \mathrm{E}-10$ & $3.49 \mathrm{E}-10$ & $1.55 \mathrm{E}-10$ & $1.98 \mathrm{E}-10$ \\
\hline $\mathrm{H} 2 \mathrm{SO} 4(\mathrm{~g})$ & 7.94E-09 & $1.38 \mathrm{E}-07$ & $1.91 \mathrm{E}-07$ & $2.84 \mathrm{E}-07$ & $3.58 \mathrm{E}-07$ & $3.91 \mathrm{E}-07$ & $1.34 \mathrm{E}-07$ & $2.42 \mathrm{E}-07$ \\
\hline $\mathrm{OClO}(\mathrm{g})$ & $9.98 \mathrm{E}-14$ & $9.27 \mathrm{E}-14$ & $2.22 \mathrm{E}-13$ & $6.92 \mathrm{E}-13$ & $1.61 \mathrm{E}-12$ & $2.60 \mathrm{E}-12$ & $1.05 \mathrm{E}-11$ & 8.99E-12 \\
\hline $\mathrm{OH}(\mathrm{g})$ & $1.73 \mathrm{E}-06$ & 7.27E-06 & 8.62E-06 & $=-05$ & $4 \mathrm{E}-05$ & $1.33 \mathrm{E}-05$ & $1.24 \mathrm{E}-05$ & $1.38 \mathrm{E}-05$ \\
\hline $\mathrm{H} 2 \mathrm{O} 2(\mathrm{~g})$ & $1.06 \mathrm{E}-10$ & $1.87 \mathrm{E}-09$ & 2.63E-09 & $4.04 \mathrm{E}-09$ & $5.42 \mathrm{E}-09$ & $6.28 \mathrm{E}-09$ & 5.39E-09 & $6.75 \mathrm{E}-09$ \\
\hline $\mathrm{HO} 2(\mathrm{~g})$ & $9.80 \mathrm{E}-11$ & 7.34E-09 & $1.24 \mathrm{E}-08$ & $2.43 \mathrm{E}-08$ & $3.99 \mathrm{E}-08$ & $5.27 \mathrm{E}-08$ & $1.02 \mathrm{E}-07$ & $9.99 \mathrm{E}-08$ \\
\hline $\mathrm{O}(\mathrm{g})$ & $2.73 \mathrm{E}-10$ & $4.88 \mathrm{E}-09$ & $6.93 \mathrm{E}-09$ & $1.10 \mathrm{E}-08$ & $1.56 \mathrm{E}-08$ & $1.91 \mathrm{E}-08$ & 3.99E-08 & $3.49 \mathrm{E}-08$ \\
\hline $\mathrm{NO}(\mathrm{g})$ & $0.00 \mathrm{E}+00$ & $3.56 \mathrm{E}-06$ & 7.15E-06 & $1.79 \mathrm{E}-05$ & $3.59 \mathrm{E}-05$ & $5.40 \mathrm{E}-05$ & $2.35 \mathrm{E}-04$ & $1.80 \mathrm{E}-04$ \\
\hline $\mathrm{NO} 2(\mathrm{~g})$ & $0.00 \mathrm{E}+00$ & 4.34E-09 & $1.24 \mathrm{E}-08$ & 4.93E-08 & $1.40 \mathrm{E}-07$ & $2.58 \mathrm{E}-07$ & $2.34 \mathrm{E}-06$ & 1.57E-06 \\
\hline $\mathrm{NO} 3(\mathrm{~g})$ & $0.00 \mathrm{E}+00$ & $1.30 \mathrm{E}-16$ & $5.29 \mathrm{E}-16$ & $3.34 \mathrm{E}-15$ & $1.34 \mathrm{E}-14$ & $3.03 \mathrm{E}-14$ & $5.75 \mathrm{E}-13$ & $3.38 \mathrm{E}-13$ \\
\hline HNO3 $(\mathrm{g})$ & $0.00 \mathrm{E}+00$ & $5.18 \mathrm{E}-14$ & $1.75 \mathrm{E}-13$ & $8.66 \mathrm{E}-13$ & $2.85 \mathrm{E}-12$ & $5.64 \mathrm{E}-12$ & $4.75 \mathrm{E}-11$ & 3.57E-11 \\
\hline $\mathrm{N} 2 \mathrm{O} 5(\mathrm{~g})$ & $0.00 \mathrm{E}+00$ & $1.75 \mathrm{E}-28$ & $2.03 \mathrm{E}-27$ & $5.09 \mathrm{E}-26$ & $5.81 \mathrm{E}-25$ & $2.41 \mathrm{E}-24$ & $4.16 \mathrm{E}-22$ & $1.65 \mathrm{E}-22$ \\
\hline $\mathrm{N} 2 \mathrm{O}(\mathrm{g})$ & $0.00 \mathrm{E}+00$ & $2.18 \mathrm{E}-11$ & $6.19 \mathrm{E}-11$ & $2.46 \mathrm{E}-10$ & $6.96 \mathrm{E}-10$ & $1.28 \mathrm{E}-09$ & $1.16 \mathrm{E}-08$ & 7.80E-09 \\
\hline $\mathrm{HNO}(\mathrm{g})$ & $0.00 E+00$ & $1.13 \mathrm{E}-12$ & $1.90 \mathrm{E}-12$ & $3.72 \mathrm{E}-12$ & $6.09 \mathrm{E}-12$ & $8.03 \mathrm{E}-12$ & $1.55 \mathrm{E}-11$ & $1.52 \mathrm{E}-11$ \\
\hline $\mathrm{HNO} 2(\mathrm{~g})$ & $0.00 \mathrm{E}+00$ & $3.00 \mathrm{E}-10$ & 7.15E-10 & $2.23 \mathrm{E}-09$ & $5.16 \mathrm{E}-09$ & $8.34 \mathrm{E}-09$ & $3.36 \mathrm{E}-08$ & $2.89 \mathrm{E}-08$ \\
\hline
\end{tabular}




\begin{tabular}{|c|c|c|c|c|c|c|c|c|}
\hline $\operatorname{Ar}(\mathrm{g})$ & $0.00 \mathrm{E}+00$ & $1.00 \mathrm{E}-04$ & $2.00 \mathrm{E}-04$ & $5.00 \mathrm{E}-04$ & $1.00 \mathrm{E}-03$ & $1.50 \mathrm{E}-03$ & $6.50 \mathrm{E}-03$ & $5.00 \mathrm{E}-03$ \\
\hline $\mathrm{BrBrO}(\mathrm{g})$ & $4.38 \mathrm{E}-21$ & $1.05 \mathrm{E}-18$ & 1.97E-18 & 4.35E-18 & $7.43 \mathrm{E}-18$ & $9.79 \mathrm{E}-18$ & $9.34 \mathrm{E}-18$ & $1.28 \mathrm{E}-17$ \\
\hline $\mathrm{BrF}(\mathrm{g})$ & $9.89 \mathrm{E}-15$ & $1.52 \mathrm{E}-13$ & $2.08 \mathrm{E}-13$ & $3.04 \mathrm{E}-13$ & $3.86 \mathrm{E}-13$ & $4.31 \mathrm{E}-13$ & $2.71 \mathrm{E}-13$ & $3.78 \mathrm{E}-13$ \\
\hline $\mathrm{BrOBr}(\mathrm{g})$ & $9.20 \mathrm{E}-20$ & $2.20 \mathrm{E}-17$ & 4.14E-17 & $9.14 \mathrm{E}-17$ & $1.56 \mathrm{E}-16$ & $2.06 \mathrm{E}-16$ & $1.96 \mathrm{E}-16$ & $2.68 \mathrm{E}-16$ \\
\hline $\mathrm{BrOO}(\mathrm{g})$ & $6.85 \mathrm{E}-17$ & $7.98 \mathrm{E}-14$ & $1.85 \mathrm{E}-13$ & $5.50 \mathrm{E}-13$ & $1.21 \mathrm{E}-12$ & $1.89 \mathrm{E}-12$ & $5.57 \mathrm{E}-12$ & $5.33 \mathrm{E}-12$ \\
\hline $\mathrm{CH} 3(\mathrm{~g})$ & $4.53 \mathrm{E}-27$ & $1.84 \mathrm{E}-31$ & $5.26 \mathrm{E}-32$ & $9.68 \mathrm{E}-33$ & $2.50 \mathrm{E}-33$ & $1.06 \mathrm{E}-33$ & $8.87 \mathrm{E}-36$ & $3.43 \mathrm{E}-35$ \\
\hline $\mathrm{Cl} 2 \mathrm{O}(\mathrm{g})$ & $1.45 \mathrm{E}-17$ & 4.57E-15 & $9.14 \mathrm{E}-15$ & $2.22 \mathrm{E}-14$ & $4.22 \mathrm{E}-14$ & $5.98 \mathrm{E}-14$ & $1.07 \mathrm{E}-13$ & 1.17E-13 \\
\hline $\mathrm{ClClO}(\mathrm{g})$ & $0.00 \mathrm{E}+00$ & $1.39 \mathrm{E}-14$ & $2.77 \mathrm{E}-14$ & $6.74 \mathrm{E}-14$ & $1.28 \mathrm{E}-13$ & $1.82 \mathrm{E}-13$ & $3.25 \mathrm{E}-13$ & $3.56 \mathrm{E}-13$ \\
\hline $\mathrm{CIF}(\mathrm{g})$ & $2.30 \mathrm{E}-14$ & $4.05 \mathrm{E}-13$ & $5.71 \mathrm{E}-13$ & 8.77E-13 & $1.18 \mathrm{E}-12$ & $1.36 \mathrm{E}-12$ & 1.17E-12 & $1.46 \mathrm{E}-12$ \\
\hline $\mathrm{ClO} 2(\mathrm{~g})$ & 3.34E-17 & 4.47E-14 & $1.07 \mathrm{E}-13$ & 3.33E-13 & $7.74 \mathrm{E}-13$ & $1.25 \mathrm{E}-12$ & $5.06 \mathrm{E}-12$ & $4.33 \mathrm{E}-12$ \\
\hline $\mathrm{ClOCl}(\mathrm{g})$ & $4.38 \mathrm{E}-17$ & $1.38 \mathrm{E}-14$ & $2.76 \mathrm{E}-14$ & 6.70E-14 & $1.27 \mathrm{E}-13$ & $1.81 \mathrm{E}-13$ & $3.24 \mathrm{E}-13$ & $3.54 \mathrm{E}-13$ \\
\hline $\mathrm{ClOO}(\mathrm{g})$ & $3.30 \mathrm{E}-16$ & $4.41 \mathrm{E}-13$ & $1.06 \mathrm{E}-12$ & 3.29E-12 & $7.65 \mathrm{E}-12$ & $1.24 \mathrm{E}-11$ & $5.00 \mathrm{E}-11$ & $4.28 \mathrm{E}-11$ \\
\hline $\mathrm{COCl}(\mathrm{g})$ & $1.46 \mathrm{E}-14$ & $3.40 \mathrm{E}-15$ & $2.81 \mathrm{E}-15$ & $2.13 \mathrm{E}-15$ & $1.65 \mathrm{E}-15$ & $1.37 \mathrm{E}-15$ & $2.53 \mathrm{E}-16$ & $4.58 \mathrm{E}-16$ \\
\hline $\mathrm{COCl} 2(\mathrm{~g})$ & 8.15E-17 & $7.98 \mathrm{E}-17$ & $7.85 \mathrm{E}-17$ & 7.34E-17 & $6.58 \mathrm{E}-17$ & $5.88 \mathrm{E}-17$ & $1.01 \mathrm{E}-17$ & $2.04 \mathrm{E}-17$ \\
\hline $\mathrm{COOH}(\mathrm{g})$ & $1.91 \mathrm{E}-13$ & $4.45 \mathrm{E}-14$ & $3.68 \mathrm{E}-14$ & 2.79E-14 & $2.16 \mathrm{E}-14$ & $1.80 \mathrm{E}-14$ & $3.32 \mathrm{E}-15$ & $6.02 \mathrm{E}-15$ \\
\hline $\cos (g)$ & $1.40 \mathrm{E}-14$ & $2.40 \mathrm{E}-18$ & 8.17E-19 & $1.92 \mathrm{E}-19$ & $5.99 \mathrm{E}-20$ & $2.91 \mathrm{E}-20$ & $5.31 \mathrm{E}-22$ & $.61 \mathrm{E}-21$ \\
\hline $\mathrm{CS}(\mathrm{g})$ & $6.48 \mathrm{E}-25$ & $6.24 \mathrm{E}-30$ & $1.50 \mathrm{E}-30$ & $2.21 \mathrm{E}-31$ & $4.88 \mathrm{E}-32$ & $1.93 \mathrm{E}-32$ & $1.69 \mathrm{E}-34$ & $5.86 \mathrm{E}-34$ \\
\hline $\mathrm{CS} 2(\mathrm{~g})$ & $1.04 \mathrm{E}-27$ & $3.10 \mathrm{E}-35$ & $3.62 E-36$ & $1.00 \mathrm{E}-36$ & $1.00 \mathrm{E}-36$ & $1.00 \mathrm{E}-36$ & $1.00 \mathrm{E}-36$ & $1.00 \mathrm{E}-36$ \\
\hline $\mathrm{H} 2 \mathrm{~S}(\mathrm{~g})$ & $5.56 \mathrm{E}-12$ & $9.56 \mathrm{E}-16$ & $3.25 \mathrm{E}-16$ & 7.63E-17 & $2.38 \mathrm{E}-17$ & $1.15 \mathrm{E}-17$ & $2.08 \mathrm{E}-19$ & 6.37E-19 \\
\hline $\mathrm{H} 2 \mathrm{~S} 2(\mathrm{~g})$ & $3.71 \mathrm{E}-21$ & $1.97 \mathrm{E}-27$ & $3.28 \mathrm{E}-28$ & $2.95 \mathrm{E}-29$ & $4.31 \mathrm{E}-30$ & $1.31 \mathrm{E}-30$ & $2.16 \mathrm{E}-33$ & $1.24 \mathrm{E}-32$ \\
\hline $\mathrm{HClCO}(\mathrm{g})$ & 7.97E-17 & $4.38 \mathrm{E}-18$ & $3.03 \mathrm{E}-18$ & $1.79 \mathrm{E}-18$ & $1.13 \mathrm{E}-18$ & 8.26E-19 & $6.77 \mathrm{E}-20$ & $1.57 \mathrm{E}-19$ \\
\hline $\mathrm{HCN}(\mathrm{g})$ & $0.00 \mathrm{E}+00$ & $1.72 \mathrm{E}-23$ & $9.98 \mathrm{E}-24$ & 4.75E-24 & $2.59 \mathrm{E}-24$ & $1.76 \mathrm{E}-24$ & $1.55 \mathrm{E}-25$ & $3.21 \mathrm{E}-25$ \\
\hline $\mathrm{HCO}(\mathrm{g})$ & $E-17$ & 19 & $=-19$ & $=-20$ & -20 & $=-20$ & -21 & $1 \mathrm{E}-21$ \\
\hline $\mathrm{HCOOH}(\mathrm{g})$ & $9.24 \mathrm{E}-13$ & $5.07 \mathrm{E}-14$ & $3.50 \mathrm{E}-14$ & $2.08 \mathrm{E}-14$ & $1.32 \mathrm{E}-14$ & $9.59 \mathrm{E}-15$ & $7.87 \mathrm{E}-16$ & $1.82 \mathrm{E}-15$ \\
\hline $\mathrm{HIO}(\mathrm{g})$ & 2.15E-09 & $\mathrm{E}-09$ & $\mathrm{E}-08$ & $E-08$ & 1.39E-08 & $1 \mathrm{E}-08$ & $3 \mathrm{E}-09$ & $3 \mathrm{E}-09$ \\
\hline $\mathrm{HNCO}(\mathrm{g})$ & $0.00 \mathrm{E}+00$ & $1.15 \mathrm{E}-18$ & $9.47 \mathrm{E}-19$ & 7.15E-19 & $5.53 \mathrm{E}-19$ & 4.59E-19 & $8.46 \mathrm{E}-20$ & $1.54 \mathrm{E}-19$ \\
\hline $\mathrm{HS}(\mathrm{g})$ & $5.29 \mathrm{E}-13$ & $3.86 \mathrm{E}-16$ & $1.57 \mathrm{E}-16$ & 4.72E-17 & $1.80 \mathrm{E}-17$ & $9.94 \mathrm{E}-18$ & 4.04E-19 & $9.69 \mathrm{E}-19$ \\
\hline $\mathrm{HSO} 3 \mathrm{Cl}(\mathrm{g})$ & $3.23 \mathrm{E}-12$ & 5.61E-11 & 7.77E-11 & $1.15 \mathrm{E}-10$ & $1.45 \mathrm{E}-10$ & $1.59 \mathrm{E}-10$ & $5.44 \mathrm{E}-11$ & $9.78 \mathrm{E}-11$ \\
\hline $\mathrm{HSO} 3 \mathrm{~F}(\mathrm{~g})$ & $2.30 \mathrm{E}-12$ & $3.99 \mathrm{E}-11$ & $5.54 \mathrm{E}-11$ & $8.24 \mathrm{E}-11$ & $1.04 \mathrm{E}-10$ & $1.13 \mathrm{E}-10$ & $3.89 \mathrm{E}-11$ & $7.00 \mathrm{E}-11$ \\
\hline $\mathrm{IBr}(\mathrm{g})$ & $0.00 \mathrm{E}+00$ & $1.36 \mathrm{E}-10$ & $1.55 \mathrm{E}-10$ & $1.76 \mathrm{E}-10$ & $1.82 \mathrm{E}-10$ & $1.78 \mathrm{E}-10$ & 4.99E-11 & $8.86 \mathrm{E}-11$ \\
\hline $\mathrm{ICl}(\mathrm{g})$ & $1.74 \mathrm{E}-09$ & 7.34E-09 & 8.61E-09 & $1.03 \mathrm{E}-08$ & $1.12 \mathrm{E}-08$ & $1.14 \mathrm{E}-08$ & 4.37E-09 & $6.94 \mathrm{E}-09$ \\
\hline IF(g) & $3.13 \mathrm{E}-13$ & $1.32 \mathrm{E}-12$ & $1.55 \mathrm{E}-12$ & $1.86 \mathrm{E}-12$ & $2.03 \mathrm{E}-12$ & $2.06 \mathrm{E}-12$ & $7.92 \mathrm{E}-13$ & $1.26 \mathrm{E}-12$ \\
\hline $100(\mathrm{~g})$ & $3.56 \mathrm{E}-17$ & $1.14 \mathrm{E}-14$ & $2.27 \mathrm{E}-14$ & $5.52 \mathrm{E}-14$ & $1.04 \mathrm{E}-13$ & $1.48 \mathrm{E}-13$ & $2.68 \mathrm{E}-13$ & $2.91 \mathrm{E}-13$ \\
\hline $\mathrm{N}(\mathrm{g})$ & $0.00 \mathrm{E}+00$ & $5.80 \mathrm{E}-18$ & $8.20 \mathrm{E}-18$ & $1.30 \mathrm{E}-17$ & $1.83 \mathrm{E}-17$ & $2.25 \mathrm{E}-17$ & $4.68 \mathrm{E}-17$ & 4.10E-17 \\
\hline $\mathrm{N} 2 \mathrm{O} 2(\mathrm{~g})$ & $0.00 \mathrm{E}+00$ & 18 & 8 & 17 & -16 & -16 & 15 & $\mathrm{E}-15$ \\
\hline $\mathrm{N} 2 \mathrm{O} 3(\mathrm{~g})$ & $0.00 \mathrm{E}+00$ & $4.72 \mathrm{E}-20$ & $2.71 \mathrm{E}-19$ & $2.70 \mathrm{E}-18$ & $1.54 \mathrm{E}-17$ & $4.25 \mathrm{E}-17$ & $1.68 \mathrm{E}-15$ & $8.66 \mathrm{E}-16$ \\
\hline $\mathrm{NBr}(\mathrm{g})$ & $0.00 \mathrm{E}+00$ & $3.68 \mathrm{E}-17$ & $5.99 \mathrm{E}-17$ & $1.12 \mathrm{E}-16$ & $1.74 \mathrm{E}-16$ & $2.20 \mathrm{E}-16$ & $3.10 \mathrm{E}-16$ & $3.40 \mathrm{E}-16$ \\
\hline $\mathrm{NH} 2(\mathrm{~g})$ & $0.00 \mathrm{E}+00$ & $1.74 \mathrm{E}-17$ & $1.72 \mathrm{E}-17$ & $1.66 \mathrm{E}-17$ & $1.57 \mathrm{E}-17$ & $1.48 \mathrm{E}-17$ & $6.05 \mathrm{E}-18$ & $8.68 \mathrm{E}-18$ \\
\hline $\mathrm{NH} 2 \mathrm{OH}(\mathrm{g})$ & $0.00 \mathrm{E}+00$ & $8.53 \mathrm{E}-19$ & $9.97 \mathrm{E}-19$ & 1.19E-18 & $1.31 \mathrm{E}-18$ & $1.33 \mathrm{E}-18$ & $5.04 \mathrm{E}-19$ & 8.09E-19 \\
\hline $\mathrm{NH} 3(\mathrm{~g})$ & $0.00 \mathrm{E}+00$ & $1.40 \mathrm{E}-14$ & $1.15 \mathrm{E}-14$ & 8.69E-15 & $6.72 \mathrm{E}-15$ & 5.56E-15 & $1.01 \mathrm{E}-15$ & $1.85 \mathrm{E}-15$ \\
\hline $\mathrm{NO} 2 \mathrm{Cl}(\mathrm{g})$ & $0.00 \mathrm{E}+00$ & $1.64 \mathrm{E}-15$ & $5.54 \mathrm{E}-15$ & $2.73 \mathrm{E}-14$ & 8.97E-14 & $1.78 \mathrm{E}-13$ & $1.50 \mathrm{E}-12$ & $1.12 \mathrm{E}-12$ \\
\hline $\mathrm{NOBr}(\mathrm{g})$ & $0.00 \mathrm{E}+00$ & $5.88 \mathrm{E}-13$ & $1.36 \mathrm{E}-12$ & 4.02E-12 & $8.84 \mathrm{E}-12$ & $1.38 \mathrm{E}-11$ & $4.05 \mathrm{E}-11$ & $3.88 \mathrm{E}-11$ \\
\hline $\mathrm{NOCl}(\mathrm{g})$ & $0.00 \mathrm{E}+00$ & $5.82 \mathrm{E}-11$ & $1.39 \mathrm{E}-10$ & $4.31 \mathrm{E}-10$ & $9.99 \mathrm{E}-10$ & $1.61 \mathrm{E}-09$ & $6.50 \mathrm{E}-09$ & 5.57E-09 \\
\hline NOF(g) & $0.00 \mathrm{E}+00$ & $1.18 \mathrm{E}-14$ & $2.80 \mathrm{E}-14$ & 8.73E-14 & $2.02 \mathrm{E}-13$ & $3.27 \mathrm{E}-13$ & $1.32 \mathrm{E}-12$ & 1.13E-12 \\
\hline $\mathrm{NOI}(\mathrm{g})$ & $0.00 \mathrm{E}+00$ & $1.34 \mathrm{E}-14$ & $2.67 \mathrm{E}-14$ & 6.46E-14 & $1.22 \mathrm{E}-13$ & $1.73 \mathrm{E}-13$ & $3.11 \mathrm{E}-13$ & $3.39 \mathrm{E}-13$ \\
\hline $\mathrm{NS}(\mathrm{g})$ & $0.00 \mathrm{E}+00$ & $1.82 \mathrm{E}-19$ & $1.26 \mathrm{E}-19$ & 7.64E-20 & $5.05 \mathrm{E}-20$ & $3.88 \mathrm{E}-20$ & 7.39E-21 & $1.22 \mathrm{E}-20$ \\
\hline
\end{tabular}




\begin{tabular}{|c|c|c|c|c|c|c|c|c|}
\hline $\mathrm{OBrO}(\mathrm{g})$ & $1.33 \mathrm{E}-19$ & $1.55 \mathrm{E}-16$ & $3.60 \mathrm{E}-16$ & 1.07E-15 & $2.35 \mathrm{E}-15$ & $3.67 \mathrm{E}-15$ & $1.08 \mathrm{E}-14$ & $1.04 \mathrm{E}-14$ \\
\hline $\mathrm{OIO}(\mathrm{g})$ & $1.05 \mathrm{E}-19$ & 3.37E-17 & $6.71 \mathrm{E}-17$ & 1.63E-16 & $3.09 \mathrm{E}-16$ & 4.37E-16 & $7.92 \mathrm{E}-16$ & $8.62 \mathrm{E}-16$ \\
\hline$S(g)$ & $3.84 \mathrm{E}-14$ & $1.19 \mathrm{E}-16$ & $5.80 \mathrm{E}-17$ & $2.23 \mathrm{E}-17$ & $1.04 \mathrm{E}-17$ & $6.54 \mathrm{E}-18$ & $5.98 \mathrm{E}-19$ & $1.12 \mathrm{E}-18$ \\
\hline S2(g) & $4.46 \mathrm{E}-16$ & 4.27E-21 & $1.02 \mathrm{E}-21$ & $1.50 \mathrm{E}-22$ & $3.28 \mathrm{E}-23$ & 1.29E-23 & $1.08 \mathrm{E}-25$ & $3.82 \mathrm{E}-25$ \\
\hline $\mathrm{S} 2 \mathrm{Br} 2(\mathrm{~g})$ & $2.58 \mathrm{E}-27$ & 3.32E-31 & $1.04 \mathrm{E}-31$ & $2.15 \mathrm{E}-32$ & $5.64 \mathrm{E}-33$ & $2.39 \mathrm{E}-33$ & $9.15 E-36$ & $5.04 \mathrm{E}-35$ \\
\hline $\mathrm{S} 2 \mathrm{Cl}(\mathrm{g})$ & $2.64 \mathrm{E}-20$ & 1.07E-24 & $3.01 \mathrm{E}-25$ & $5.50 \mathrm{E}-26$ & $1.39 \mathrm{E}-26$ & $5.89 \mathrm{E}-27$ & 4.57E-29 & $1.80 \mathrm{E}-28$ \\
\hline $\mathrm{S} 2 \mathrm{Cl} 2$ (g) & $5.62 \mathrm{E}-24$ & $9.52 \mathrm{E}-28$ & $3.19 \mathrm{E}-28$ & 7.21E-29 & $2.11 \mathrm{E}-29$ & $9.63 \mathrm{E}-30$ & $6.92 \mathrm{E}-32$ & $3.05 \mathrm{E}-31$ \\
\hline $\mathrm{S} 2 \mathrm{O}(\mathrm{g})$ & $5.38 \mathrm{E}-15$ & $9.19 \mathrm{E}-19$ & $3.11 \mathrm{E}-19$ & $7.28 \mathrm{E}-20$ & $2.25 \mathrm{E}-20$ & $1.09 \mathrm{E}-20$ & $1.90 \mathrm{E}-22$ & $5.88 \mathrm{E}-22$ \\
\hline $\mathrm{S} 3(\mathrm{~g})$ & $3.22 \mathrm{E}-25$ & $9.57 \mathrm{E}-33$ & $1.11 \mathrm{E}-33$ & $6.30 \mathrm{E}-35$ & $6.43 \mathrm{E}-36$ & $1.59 \mathrm{E}-36$ & $1.00 \mathrm{E}-36$ & $1.00 \mathrm{E}-36$ \\
\hline $\mathrm{S} 4(\mathrm{~g})$ & $2.89 \mathrm{E}-34$ & $1.00 \mathrm{E}-36$ & $1.00 \mathrm{E}-36$ & $1.00 \mathrm{E}-36$ & $1.00 \mathrm{E}-36$ & $1.00 \mathrm{E}-36$ & $1.00 \mathrm{E}-36$ & $1.00 \mathrm{E}-36$ \\
\hline $\mathrm{S} 5(\mathrm{~g})$ & $1.00 \mathrm{E}-36$ & $1.00 \mathrm{E}-36$ & $1.00 \mathrm{E}-36$ & $1.00 \mathrm{E}-36$ & $1.00 \mathrm{E}-36$ & $1.00 \mathrm{E}-36$ & $1.00 \mathrm{E}-36$ & $1.00 \mathrm{E}-36$ \\
\hline S6(g) & $E-36$ & $\mathrm{E}-36$ & $\mathrm{E}-36$ & $E-36$ & E-36 & E-36 & $1.00 \mathrm{E}-36$ & $.00 \mathrm{E}-36$ \\
\hline $\mathrm{SBr} 2(\mathrm{~g})$ & $2.54 \mathrm{E}-17$ & $1.06 \mathrm{E}-18$ & $6.82 \mathrm{E}-19$ & $3.65 \mathrm{E}-19$ & $2.05 \mathrm{E}-19$ & 1.39E-19 & 5.79E-21 & $1.70 \mathrm{E}-20$ \\
\hline $\mathrm{SCl}(\mathrm{g})$ & $2.57 \mathrm{E}-15$ & 3.34E-17 & $1.93 \mathrm{E}-17$ & $9.20 \mathrm{E}-18$ & $4.98 \mathrm{E}-18$ & $3.36 \mathrm{E}-18$ & $2.85 \mathrm{E}-19$ & $5.98 \mathrm{E}-19$ \\
\hline $\mathrm{SCl} 2(\mathrm{~g})$ & $1.44 \mathrm{E}-15$ & $7.88 \mathrm{E}-17$ & $5.41 \mathrm{E}-17$ & $3.19 \mathrm{E}-17$ & $2.00 \mathrm{E}-17$ & $1.45 \mathrm{E}-17$ & $1.14 \mathrm{E}-18$ & $2.67 \mathrm{E}-18$ \\
\hline $\mathrm{SF}(\mathrm{g})$ & $7.85 \mathrm{E}-18$ & $1.02 \mathrm{E}-19$ & $5.91 \mathrm{E}-20$ & $2.82 \mathrm{E}-20$ & $1.52 \mathrm{E}-20$ & $1.03 \mathrm{E}-20$ & 8.74E-22 & $1.84 \mathrm{E}-21$ \\
\hline $\mathrm{SO}(\mathrm{g})$ & $2.72 \mathrm{E}-08$ & $1.50 \mathrm{E}-09$ & $1.04 \mathrm{E}-09$ & $6.34 \mathrm{E}-10$ & 4.20E-10 & $3.23 \mathrm{E}-10$ & $6.18 \mathrm{E}-11$ & $1.02 \mathrm{E}-10$ \\
\hline $\mathrm{SO} 2 \mathrm{Cl} 2(\mathrm{~g})$ & $5.82 \mathrm{E}-16$ & $1.01 \mathrm{E}-14$ & $1.40 \mathrm{E}-14$ & $2.08 \mathrm{E}-14$ & $2.61 \mathrm{E}-14$ & $2.85 \mathrm{E}-14$ & $9.78 \mathrm{E}-15$ & $1.76 \mathrm{E}-14$ \\
\hline $\mathrm{SO} 2 \mathrm{CIF}(\mathrm{g})$ & $5.95 \mathrm{E}-16$ & $1.03 \mathrm{E}-14$ & $1.43 \mathrm{E}-14$ & $2.13 \mathrm{E}-14$ & $2.68 \mathrm{E}-14$ & $2.92 \mathrm{E}-14$ & $1.01 \mathrm{E}-14$ & $1.81 \mathrm{E}-14$ \\
\hline $\mathrm{SO} 3(\mathrm{~g})$ & $5.66 \mathrm{E}-06$ & $9.94 \mathrm{E}-05$ & $1.39 \mathrm{E}-04$ & $2.13 \mathrm{E}-04$ & $2.83 \mathrm{E}-04$ & $3.28 \mathrm{E}-04$ & $2.74 \mathrm{E}-04$ & $3.45 \mathrm{E}-04$ \\
\hline $\mathrm{SOCl}(\mathrm{g})$ & $7.07 \mathrm{E}-11$ & $1.64 \mathrm{E}-11$ & $1.35 \mathrm{E}-11$ & $1.02 \mathrm{E}-11$ & $7.80 \mathrm{E}-12$ & $6.46 \mathrm{E}-12$ & $1.14 \mathrm{E}-12$ & $2.10 \mathrm{E}-12$ \\
\hline $\mathrm{SOCl} 2(\mathrm{~g})$ & $4.44 \mathrm{E}-14$ & 4.33E-14 & 4.23E-14 & $3.95 \mathrm{E}-14$ & 3.50E-14 & $3.12 \mathrm{E}-14$ & $5.12 \mathrm{E}-15$ & $1.05 \mathrm{E}-14$ \\
\hline SOF(g) & $4.70 \mathrm{E}-11$ & $1.09 \mathrm{E}-11$ & $8.96 \mathrm{E}-12$ & $6.78 \mathrm{E}-12$ & 5.19E-12 & 4.30E-12 & 7.63E-13 & $1.40 \mathrm{E}-12$ \\
\hline
\end{tabular}

\section{References}

Roberts, T. J., Martin, R. S., and Jourdain, L.: Reactive bromine chemistry in Mount Etna's volcanic plume: The influence of total $\mathrm{Br}$, high-temperature processing, aerosol loading and plume-air mixing, Atmospheric Chemistry and Physics, 14, 11201-11219, doi:10.5194/acp-14-11201-2014, 2014. 